Review Article

\title{
Zone of Polarizing Activity Regulatory Sequence Mutations/Duplications with Preaxial Polydactyly and Longitudinal Preaxial Ray Deficiency in the Phenotype: A Review of Human Cases, Animal Models, and Insights Regarding the Pathogenesis
}

\author{
Mohammad M. Al-Qattan \\ King Saud University, Riyadh, Saudi Arabia \\ Correspondence should be addressed to Mohammad M. Al-Qattan; moqattan@hotmail.com
}

Received 9 October 2017; Revised 19 December 2017; Accepted 16 January 2018; Published 13 February 2018

Academic Editor: Thomas Lufkin

Copyright (C) 2018 Mohammad M. Al-Qattan. This is an open access article distributed under the Creative Commons Attribution License, which permits unrestricted use, distribution, and reproduction in any medium, provided the original work is properly cited.

Clinicians and scientists interested in developmental biology have viewed preaxial polydactyly (PPD) and longitudinal preaxial ray deficiency (LPAD) as two different entities. Point mutations and duplications in the zone of polarizing activity regulatory sequence (ZRS) are associated with anterior ectopic expression of Sonic Hedgehog (SHH) in the limb bud and usually result in a PPD phenotype. However, some of these mutations/duplications also have LPAD in the phenotype. This unusual PPD-LPAD association in ZRS mutations/duplications has not been specifically reviewed in the literature. The author reviews this unusual entity and gives insights regarding its pathogenesis.

\section{Introduction}

Preaxial polydactyly (PPD) indicates the presence of extra digit(s) on the preaxial side of the hand or foot [1]. Genetically, PPD is classified according to either the TemtamyMcKusick [2] or the Winter-Tickle [3] classification. The former classifies PPD into 4 types: PPD I is duplication of a biphalangeal thumb, PPD II is isolated triphalangeal thumb or thumb duplication with a triphalangeal component, PPD III is polydactyly of the index finger, and PPD IV is polysyndactyly of the thumb. The latter classification includes syndromes in which PPD is a constant feature such as the triphalangeal thumb-polysyndactyly syndrome (TPTPS, MIM 188770), Werner syndrome (Tibial Hypoplasia, Polysyndactyly Triphalangeal Thumb Syndrome or THPTTS, OMIM 188770), and Laurin-Sandrow syndrome (mirror-image polydactyly of the hands and feet, absent tibiae, and duplication of the fibulae; OMIM 135750). In 2013, it was proposed that LPAD should be included in the PPD spectrum [4]. The normal development of the thumb/big toe requires the absence of Sonic Hedgehog (SHH) activity in the most anterior part of the mesoderm, which is the area of the developing digit 1 . The normal expression/activity of $\mathrm{SHH}$ participates in the normal development of the ulna/fibula as well as digits 2-5 (Figure 1(a)). The abnormal anterior ectopic expression of SHH will result in PPD as shown in Figure 1(b) [5]. Furthermore, the degree of PPD will depend on the degree of abnormal ectopic SHH expression (duplication of a biphalangeal preaxial digit is seen with minor $\mathrm{SHH}$ ectopic expression while mirror-image PPD is seen with extreme SHH ectopic expression) [5-7].

Embryologically, the thumb/radius and big toe/tibia (also known as the preaxial rays) develop under the influence of ectodermal Fibroblast Growth Factor 8 (FGF8) and the expression of T-BOX5 (TBX5), HOX, SALL1, and SALL4 in the anterior mesoderm (Figure 1(a)). Hence, deficiency of FGF8/TBX5/SALL1\&4 leads to longitudinal preaxial ray deficiency (LPAD); and this is considered as failure of formation 

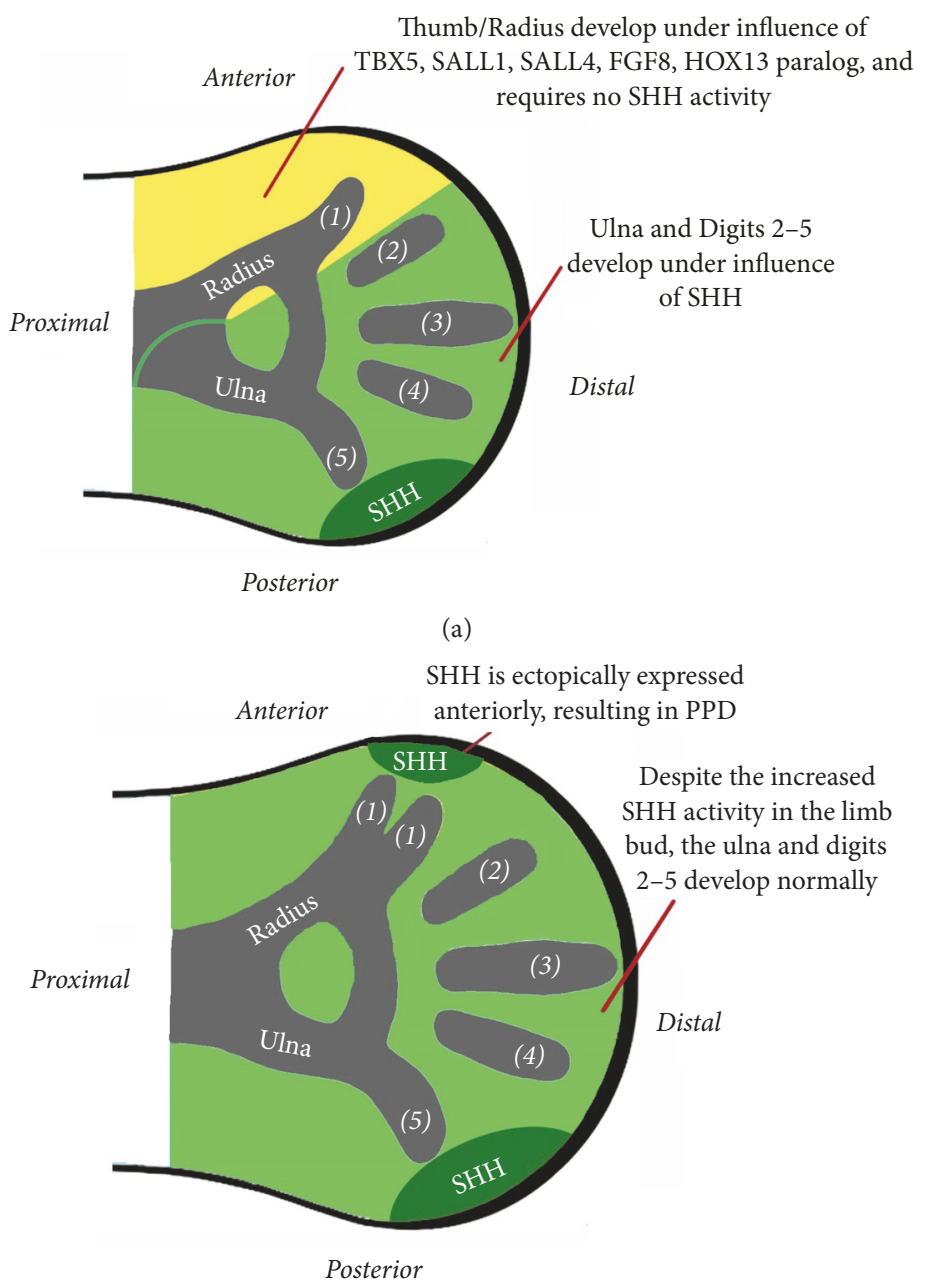

(b)

FIgURE 1: (a) SHH is posteriorly located in the Zone of Polarizing Activity (ZPA, marked with dark green). The SHH protein is a diffusible morphogen. Normally, SHH activity (light green color) extends anteriorly to reach digit 2. There is no SHH expression or activity in the zones of digit 1/radius. (b) Anterior ectopic expression of SHH results in PPD (preaxial polydactyly).

of the preaxial ray along the anteroposterior axis [8]. The spectrum of LPAD starts with isolated mild hypoplasia of the thumb/big toe and ends with complete absence of the entire preaxial ray [9].

The zone of polarizing activity regulatory sequence (ZRS) is the main controller of SHH activity in the limb bud [13]. Normally, SHH expression is restricted posteriorly within the Zone of Polarizing Activity (ZPA) and it moderates the anteroposterior axis of limb development [6]. Although the $\mathrm{SHH}$ protein diffuses in an anterior direction, it does not normally reach the most anterior part of the mesoderm where the preaxial ray develops (Figure 1(a)). The ZRS is located in humans on chromosome 7 and in mice on chromosome 5 , within intron 5 of $L M B R 1$ (about $1 \mathrm{Mb}$ telomere of the $\mathrm{SHH}$ gene). There are several point mutations/duplications in the ZRS in humans and in mice models [14]. They are all associated with anterior ectopic expression of $\mathrm{SHH}$ and result in various forms of PPD. Some of these point mutations/duplications in humans and mice models will also have LPAD in the phenotype (Table 1). In the current review, this combined phenotype will be named "PPD-LPAD association."

1.1. ZRS Point Mutations at Position 404 in Humans. Point mutations at position 404 appear to be a hotspot for PPDLPAD associations. The phenotype is known as Werner syndrome. In this syndrome, tibial hypoplasia is usually more commonly seen than hypoplasia of the radius.

One family was found to have $\mathrm{G}>\mathrm{A}$ transition at position 404 of the ZRS. All family members had PPD except the proband who also had bilateral tibial hemimelia and mild radius dysplasia [16]. Another family with 404 G>A ZRS mutation had PPD-five-fingered hand-tibial hemimelia phenotype [17].

The 404 G>T ZRS mutation was reported in one family and resulted in a variable phenotype including PPD in the hands and feet, opposable and nonopposable triphalangeal thumbs, broad big toes/first metatarsals (which may be considered as "Forme Fruste" PPD), cutaneous syndactyly, and concurrent preaxial and postaxial polydactyly in one 
TABLE 1: Human ZRS mutations/duplications in which there is PPD-LPAD association in the phenotype.

ZRS mutations/duplications

The phenotype

(A) Point mutations

ZRS $402 \mathrm{C}>\mathrm{T}$

ZRS $404 \mathrm{G}>\mathrm{A}$

Either Werner phenotype or PPD-five-fingered hand-tibial hemimelia phenotype

ZRS $404 \mathrm{G}>\mathrm{T}$

ZRS $404 \mathrm{G}>\mathrm{C}$

ZRS $406 \mathrm{~A}>\mathrm{G}$

ZRS $417 \mathrm{~A}>\mathrm{G}$

ZRS $619 \mathrm{C}>\mathrm{T}$

Werner syndrome phenotype

(B) Duplications

Microduplications $(<80 \mathrm{~kb})$ reported by Lohan et al.

[15]

96,605 bp duplication from nt $156,240,230$ to $n t$ $156,336,835$ at $7 \mathrm{q} 36.3$

Vhenotype
Variable phenotypes of PPD-LPAD association
Triphalangeal thumbs in the hands and PPD/tibial defects in the lower limbs
Werner syndrome phenotype
Mirror image polydactyly in all 4 limbs and bilateral tibial defects
PPD-LPAD associations confined to the upper limbs. The lower limbs are
normal

Laurin-Sandrow syndrome phenotype

Syndactyly type IV with concurrent tibial hypoplasia

Werner syndrome: Tibial Hypoplasia, Polysyndactyly Triphalangeal Thumb Syndrome or THPTTS, OMIM 188770; Laurin-Sandrow syndrome: mirror-image polydactyly of the hands and feet, absent tibiae, and duplication of the fibulae; OMIM 135750; Syndactyly type IV: complete syndactyly of all digits with polydactyly, OMIM 186200

case. The LPAD phenotype in the same family was also variable and included tibial hemimelia, slightly short radius, small radius and ulna, and hypoplastic radius with thumb hypoplasia. In this particular mutation, LPAD of the upper limbs was more commonly seen than LPAD of the lower limbs [18].

In contrast to the variable phenotype of the $404 \mathrm{G}>\mathrm{T}$ ZRS mutation, the $404 \mathrm{G}>\mathrm{C}$ ZRS mutation resulted in a more consistent phenotype. All affected patients had triphalangeal thumbs in the hands and PPD in the feet. Tibial defects and bowing of the fibula were a common feature. Interestingly, one patient had duplicated fibulae [16].

1.2. ZRS Point Mutations at Positions 406, 417, and 619 in Humans. The $406 \mathrm{~A}>\mathrm{G}$ ZRS mutation was reported in two families with features of Werner mesomelic syndrome (triphalangeal thumbs, PPD, and tibial defects) [19].

The ZRS $417 \mathrm{~A}>\mathrm{G}$ mutation presented with a very unique phenotype of mirror-image polydactyly of the four extremities and bilateral tibial deficiency [20]. Interestingly, the same mutation in a mosaic form resulted in isolated triphalangeal thumb or thumb duplication with a triphalangeal component (PPD type II) [20].

The ZRS $619 \mathrm{C}>\mathrm{T}$ mutation was also unique because all defects were confined to the upper limbs [21]. In the upper limbs, the PPD presented as triphalangeal thumb, polydactyly of the index finger, duplication of a biphalangeal thumb, and rudimentary PPD (i.e., a preaxial nubbin). The LPAD was represented by thumb aplasia in two family members. More interesting, a third family member had bilaterally absent thumb and complete aplasia of the radius on the right and partial aplasia of the radius on the left. The same patient had absence of the left kidney and cardiac defects. This phenotype is a VACTERL phenotype (Vertebral defects, imperforate Anus, Cardiac defects, TracheoEsophageal fistula, Renal defects, and preaxial upper Limb defects) since the patient had three out of the six VACTERL diagnostic criteria. The pathogenesis of this phenotype will be discussed in more detail later in the review.

1.3. ZRS Point Mutations at Position 402 in Humans. Previous authors have emphasized that ZRS point mutations resulting in Werner syndrome (which has PPD-LPAD association in the phenotype) have "strong" ectopic anterior SHH expression [16]. Further evidence regarding this strong anterior expression comes from appearance of mirror-image duplication of the hands and feet in some patients with ZRS point mutations [20]. Experimentally, transplantation of few $\mathrm{SHH}$ expressing cells to the anterior limb bud results in PPD. As more cells are transplanted, higher degrees of PPD are seen and eventually mirror-image polydactyly is observed [6]. Perhaps the most convincing evidence that the PPDLPAD association requires a strong $\mathrm{SHH}$ anterior expression comes from observations of the phenotypes of ZRS $402 \mathrm{C}>\mathrm{T}$ mutations in the hetero- and homozygous forms. In the heterozygous form, the mutation results in isolated PPD; and, in the homozygous form, the mutation results in a Werner syndrome phenotype [22].

1.4. Duplications Encompassing the ZRS in Humans. Most duplications encompassing the ZRS in humans result in either triphalangeal thumb-polysyndactyly syndrome (TPTPS, MIM 174500) or Haas-type polysyndactyly (also known as syndactyly type IV, MIM 186200) [15, 16, 23].

Lohan et al. [15] reported on three unrelated families with overlapping microduplications $(<80 \mathrm{~Kb})$ encompassing the ZRS resulting in Laurin-Sandrow syndrome phenotype. The fact that mirror-image duplications were seen with ZRS duplications indicated that some duplications resulted in strong SHH enhancements. Indeed, Wu et al. [24] reported on 
a Chinese family with a ZRS duplication resulting in complete syndactyly of all digits with polydactyly (syndactyly type IV, OMIM 186200) and concurrent tibial hypoplasia [24].

Our review shows that all cases with ZRS duplications show syndactyly in the phenotype; and with strong $\mathrm{SHH}$ enhancements PPD-LPAD association may also be seen.

1.5. Mice Models Involving the ZRS and Showing PPD-LPAD Association in the Phenotype. The Sasquatch (Ssq) is a mouse mutation within the ZRS and it arose through a transgenic insertion [13, 25]. Heterozygotes have isolated PPD on the hind feet only. Homozygotes have higher grades of PPD in both the forelimbs and hind limbs; and some mice also had shortening of the long bones. In the homozygous phenotype, the reduction of long bones was more prominent in the hind limbs and usually involved both the tibia and fibula.

The hemimelic extra toe ( $\mathrm{Hx})$ mouse polydactylous mutation was also mapped to the ZRS [26]. Mutants have PPD and hypoplasia of the tibia/radius. This mouse model seems to be a better corollary to the accounts reported in humans than the Ssq model because the reduction of long bones is focused on the preaxial bones only.

1.6. Why Do Mutations/Duplications of the ZRS only Affect the Limb Bud? Besides the limb bud, SHH is expressed in the brain, neural floor plate, and epithelial linings of the lung and gut. Yet, ZRS mutations/duplications have no neural or epithelial defects. The reason for that is the fact that ZRS is a limb-specific enhancer. Brain (SBE1-4) and floor plate (SFPE1, 2) enhancers are located within the $S H H$ gene. Epithelial enhancers are located in next gene (Rnf32). The $L M B R 1$ gene (where the ZRS is located) is in the next gene over [27].

1.7. The Normal Development of ZPA and SHH. The normal development of the ZPA (in which $\mathrm{SHH}$ is located) passes through 3 stages [28, 29] (Figure 2). "Initiation or prepatterning" (Figure 2(a)) establishes the normal anteroposterior polarity in the early limb bud by the normal expression of the ZPA posteriorly. This normal polarity occurs because of the antagonistic interaction between GLI3R (the repressor form of GLI3) and HAND2 in the early limb bud, resulting in the restriction of GLI3R anteriorly and HAND2 posteriorly [28].

In the "induction" stage (Figure 2(b)), the normal expression of SHH within the ZPA occurs under the influence of HAND2 and $5^{\prime}$ HOXD. These inducers are located in the posterior mesoderm of the limb bud and are known as the "positive" regulators of SHH. Loss of function of these positive regulators in experimental animals will result in a reduction of SHH activity [Trachini et al., 2006].

Finally, "maintenance/restriction/control" of SHH activity will be described separately. SHH activity within the ZPA is "maintained" by FGF4 (expressed in the posterior part of the AER) and by WNT7A (expressed in the dorsal ectoderm). SHH induces FGF4 in the overlying AER and FGF4 will then help maintain the expression of $\mathrm{SHH}$; and this is known as the SHH-FGF4 feedback loop [30, 31] (Figure 2(c)). "Restriction" of SHH (preventing its transcriptional activation at the most anterior part of the mesoderm) is mediated by the "negative" regulators of SHH: GLI3R, ALX4, TWIST1, and EVT4/5 (Figure 2(d)). This restriction of SHH expression "prevents" the formation of PPD (Figure 1(b)). Finally, the ZRS is the main "controller" of SHH activity in the limb bud as mentioned earlier in the Introduction [13]. However, other limb-specific cis-regulatory elements of $\mathrm{SHH}$ exist. Early evidence came from the fact that several families with PPD and linkage to 7q36 do not have any ZRS defects [32, 33]. More recently, Petit et al. [10] reported on a "PPD-hypertrichosis" phenotype in a French family. No pathogenic variants or copy number variations were found in the ZRS. Instead, there was loss of a 2026 bp region in the gene desert $240 \mathrm{~kb}$ from the $\mathrm{SHH}$ promotor in all affected patients. Transfection experiments identified an inhibitory cis-regulatory element that is believed to be active in the anterior margin silencing anterior expression of Shh.

\subsection{How Are Mutations/Duplications/of the ZRS Translated} into an Anterior Ectopic SHH Expression in the Limb Bud? As mentioned earlier, the $\mathrm{SHH}$ protein does not normally reach the most anterior part of the limb bud. There are two different ways by which $\mathrm{SHH}$ can reach the most anterior part of the limb bud (which results in PPD): the deficiency of $\mathrm{SHH}$ negative regulators (GLI3, ALX4, TWIST1, and ETV4/5) and the expansion of the ZPA boundary itself.

Lettice et al. [27] showed that members of two groups of ETS transcription factors act directly at the ZRS: the normal binding of GABP $\alpha /$ ETS1 at multiple sites of the ZRS regulates the ZPA boundary, while the normal binding of ETV4/5 restricts $\mathrm{SHH}$ expansion outside the ZPA. With ZPA point mutations/duplications, changes in ETS binding will lead to anterior ectopic expression of SHH and PPD [27].

1.9. How Do the Transcription Factors ETV4/5 Restrict Anterior SHH Expansion? ETV4/5 activation is dependent on the overlying fibroblast growth factors within the apical ectodermal ridge (AER). Furthermore, ETV4/5 are expressed in the distal mesoderm along the entire distal edge (just below the AER). There is sufficient genetic and biochemical evidence suggesting that the anterior restriction of $\mathrm{SHH}$ by ETV4/5 is through regulation of the dimerization of Twist1 and Hand2 [34]. Twist1 and Hand2 are members of the basic helix-loop-helix family. They antagonize each other by forming protein heterodimers. The interaction occurs at the E-box DNA sequence elements to form the heterodimer [35]. As mentioned earlier, Alx4 is a "negative" regulator of $\mathrm{SHH}$ activity. This means that it is expressed anteriorly in the mesoderm and it acts to restrict $\mathrm{SHH}$ anterior expansion. Mutations in mouse Alx4 gene cause Strong's luxoid polydactyly and anterior ectopic expression of SHH [36]. Homozygotes show a very strong anterior $\mathrm{SHH}$ expression. As expected, homozygotes have mirror-image polydactyly (the highest grade of PPD) as well as reduction of the radius and tibia (i.e., PPD-LPAD association) [37].

In contrast, Hand2 is a "positive" regulator of $\mathrm{SHH}$ activity. This means that it is expressed posteriorly in the mesoderm and helps induce posterior $\mathrm{SHH}$ expression. Hence, Hand2-deficient mice lack SHH expression [38], while misexpression of Hand2 anteriorly will induce anterior 


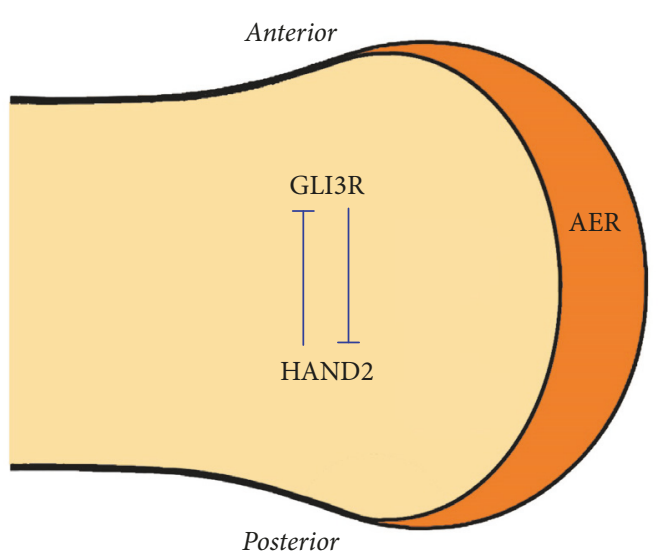

(a)

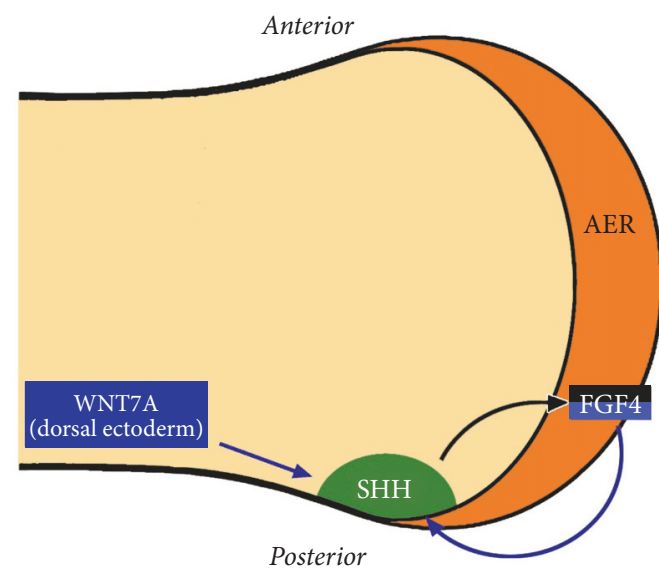

(c)

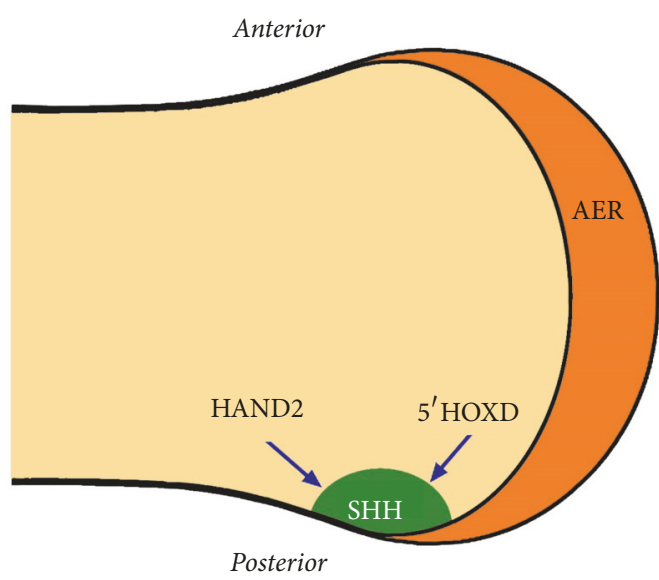

(b)

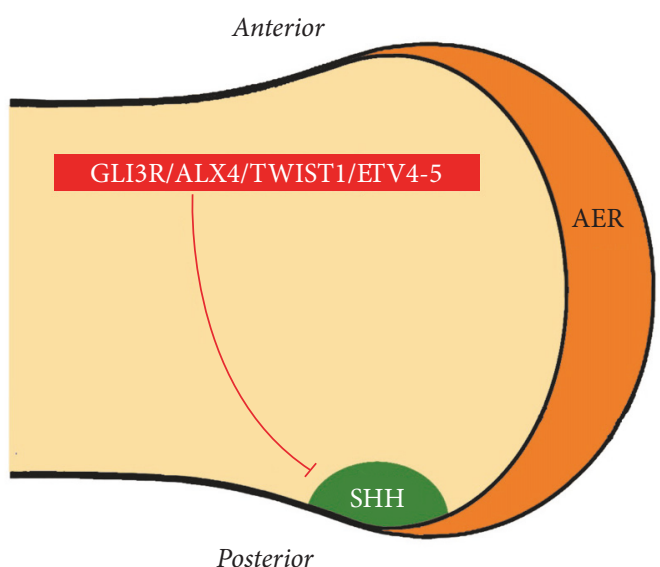

(d)

FIGURE 2: (a) Prepatterning establishes anteroposterior polarity of the early limb bud as a result of the antagonistic activity of GLI3R anteriorly and HAND2 posteriorly. This results in establishing competency for ZPA (Zone of Polarizing Activity) induction in the posterior mesoderm. (b) Next, SHH is induced within the ZPA (colored dark green) by HAND2 and 5'HOXD. (c) Following the expression of SHH, its maintenance is through WNT7A and the SHH-FGF4 feedback loop. (d) "Restriction" of SHH (preventing its transcriptional activation at the most anterior part of the mesoderm) is mediated by the "negative" regulators of SHH: GLI3R, ALX4, TWIST1, and EVT4/5. Note that other inhibitory regulatory modules exist such as the module described by Petit et al. [10].

ectopic SHH expression and mirror-image polydactyly [39, 40]. More interesting, Hand2 also binds to the ZRS [41].

\subsection{What Are the Most Prominent Molecular Changes Associ-} ated with Anterior SHH Expression? Several animal models with anterior SHH expression [36, 42-44] showed similar molecular changes in the limb bud. These changes include the abnormal anterior ectopic expression of Fgf4 in the AER and the abnormal anterior ectopic expression of $5^{\prime}$ Hox in the mesoderm of the early limb bud.

Normally, the AER has a high posterior expression of FGF4 and this is partly mediated through the SHH-FGF4 feedback loop [30]. In contrast, FGF8 is normally highly expressed along the entire AER. Animal models with ectopic anterior expression of $\mathrm{SHH}$ in the mesoderm showed the abnormal anterior expression of FGF4 in the AER [36, 4244].

The other major molecular change with anterior $\mathrm{SHH}$ expression is the abnormal HOX expression. In normal early development, HOXD are expressed in the autopod in a collinear way, with lower numbers (i.e., the $3^{\prime}$ group) expressed anteriorly and the $5^{\prime}$ group (i.e., HOXD 10-13) expressed posteriorly. This is known as the "Russian dolls" strategy of expression [45]. The second wave of HOX expression is known as the "reverse collinearity" pattern and it occurs under the influence of both $\mathrm{SHH}$ and the regulatory sequences centromeric to the HOXD cluster [45]. In this phase, HOXD 13 is transcribed all over the mesoderm (including the anterior thumb area); while HOXD 10-HOXD 12 are expressed in digits $2-5$ only. With anterior ectopic expression of Shh, animal models show anterior ectopic expression of the Hoxd13 during early development [37]. This is expected since Hoxd13 is known to be induced by Shh [46].

A third molecular change with anterior $\mathrm{SHH}$ expression is in the pattern of SALL1 expression. Normally, SALL1 expression in the mesoderm is posteriorly biased. In chick spalt homologous, anterior Shh expression leads to an expanded csall expression anteriorly [47]. This is not surprising because 


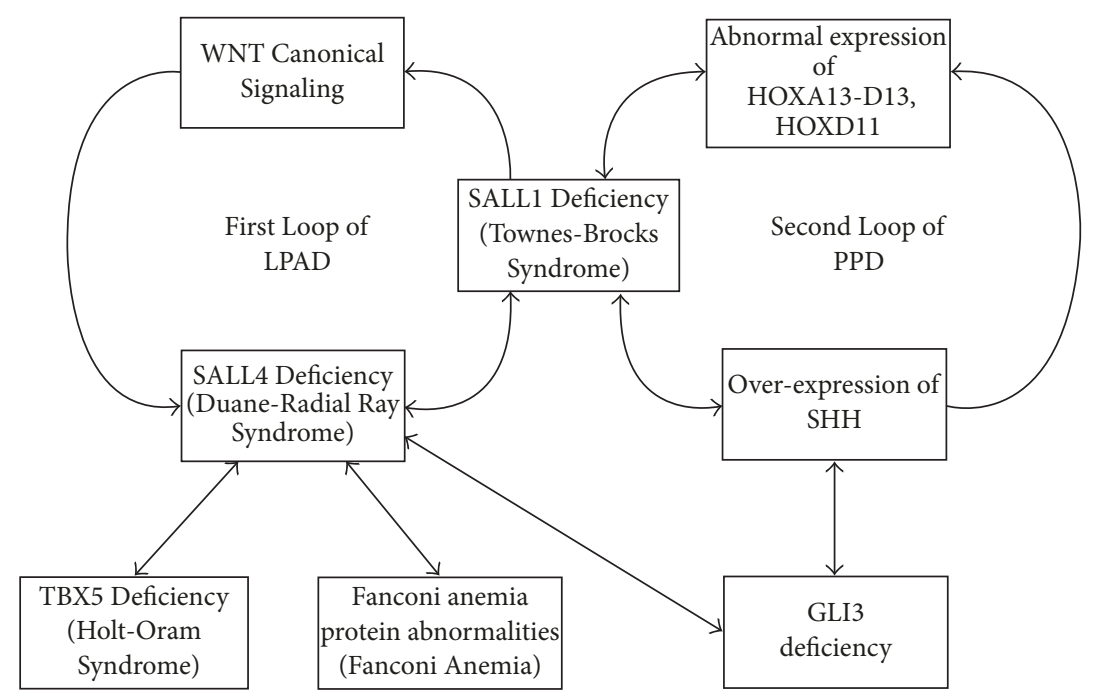

FIGURE 3: The interactions between the LPAD and PPD loops may explain the PPD-LPAD association related to ZRS mutations/duplications (see text for details). Note that both loops share SALL1 and GLI3. Deficiency of SALL1 (within the LPAD loop) will affect both the expression of SHH and the HOX system as described by Kawakami et al. [11]. Hence, the Townes-Brocks phenotype may present as LPAD only, PPD only, or PPD-LPAD association. GLI3 deficiency will result in anterior ectopic expression of SHH and PPD, since GLI3 is a negative regulator of SHH. Simultaneously, GLI3 deficiency may result in SALL4 deficiency as described by Akiyama et al. [12]. Hence, GLI3 is another important link between the two loops.

other animal models showed that Shh signaling participates in the expression of Sall1 [11].

Finally, SHH-GLI3 interactions play an important role in preaxial ray development [48]. The normal posterior $\mathrm{SHH}$ expression maintains the GLI3 transcription factor as a full-length activator (GLI3A). Anteriorly (where there is no $\mathrm{SHH}$ expression), GLI3 is processed into a repressor (GLI3R). The gradient of GLI3R transduces $\mathrm{SHH}$ signaling and disturbances of the normal SHH-GLI3 pattern affect the regulation of digit number and identity [49].

1.11. The Pathogenesis of PPD-LPAD Association in ZRS Mutations/Duplications. The molecular interactions within the mesoderm as well as mesodermal-ectodermal interactions within the developing limb bud are too complex to simply state that there is a single pathogenesis explaining the PPDLPAD association seen with strong anterior SHH expression.

One possible pathogenesis is through the interactions of two mesodermal loops shown in Figure 3. The first loop is labelled as the LPAD loop because its components are known to result in syndromes in which LPAD is a feature. The key player of the first loop is SALL 4. SALL4 interacts with TBX5 to regulate the development of the thumb, radius, and heart [50]. There is also evidence that SALL4-Fanconi anemia complementation group L interactions exist [51]. Furthermore, SALL4-GLI3 system interactions exist and the system functions upstream of the Shh-expressing ZPA and Fgf8expressing AER in both the forelimbs and hind limbs [12]. Finally, SALL4 forms a dimer with SALL1 and both cooperate in anorectal, heart, and kidney development [52]. SALL1 could then enhance the WNT canonical signaling [53] which could result in SALL4 activation via the interactions of LEF1 and TCF (both LEF1 AND TCF are WNT-related transcription factors) at the SALL4 promoter [54]. This loop explains the overlapping phenotypes of four syndromes: Duane-radial ray syndrome with SALL4 mutations (MIM 607323); Fanconi anemia (MIM 227650); Holt-Oram syndrome with TBX5 mutations (MIM142900); and Townes-Brocks syndrome with SALL1 mutations (MIM 107480). SALL1 of the LPAD loop becomes the key player of the second PPD loop. It is interesting to note that Townes-Brocks syndrome is the only LPAD syndrome in which PPD is commonly seen in the phenotype; and this is to be expected since SALL1 is the link between the two loops. SALL1 interacts with SHH and the HOX system (19]. Finally, the SHH anterior expression results in abnormal $5^{\prime} \mathrm{HOX}$ anterior expression in early development as mentioned earlier. The interactions between these two loops may explain the pathogenesis of PPD-LPAD association. These interactions are probably through SALL1 and GLI3 because both are shared by both loops (Figure 3).

A second possible pathogenesis of PPD-LPAD is through the overexpression of HOXD 11-HOXD 13 in the anterior mesoderm during early development which occurs secondary to anterior ectopic expression of SHH. In an animal model, Yang et al. [7] showed that the anterior ectopic expression of Shh results in an anterior ectopic expression of Hoxd 11-Hoxd 13. Zákány et al. [55] showed that the ectopic expression of Hoxd 11-Hoxd 13 in experimental animals was associated with Shh anterior ectopic expression and the phenotype varied among animals to include PPD only, mirror-image polydactyly with double ulnae, and LPAD (the latter was manifested by loss of the preaxial digit). This phenotypic variation is very similar to the various phenotypes of ZRS mutations/duplications, including the PPD-LPAD 
association. However, the ectopic expression of $5^{\prime} \mathrm{HOX}$ is upstream of SHH and, hence, it does not fully explain the downstream pathogenesis of PPD-LPAD association.

1.12. How Can We Explain the VACTERL Phenotype with ZRS Mutations? Al-Qattan et al. [21] showed that ZRS 619 C>T mutation may result in a VACTERL phenotype (without PPD). Most VACTERL cases are sporadic and their genetic bases are unknown [56]. Animal models link the VACTERL phenotype to Shh signaling [57]. In humans, mutations and deletions of FOXF1 (which is linked to SHH signaling) result in a VACTERL phenotype [58]. Furthermore, HOXD13 mutations may result in a VACTERL phenotype; and, as mentioned earlier, HOXD13 is a downstream target of $\mathrm{SHH}$ [59]. All these cases as well as the case reported by Al-Qattan et al. [21] link the SHH signaling to at least some cases of the VACTERL phenotype. The VACTERL phenotype in ZRS mutations may also be explained by the interactions of SHH with the LPAD loop in Figure 1. SHH gene mutation analysis was previously reported to be negative in patients with VACTERL phenotype [60]. Our review suggests that screening for ZRS mutations and other genes related to $\mathrm{SHH}$ signaling (such as FOXF1, HOXD 13, GLI 2, and GLI 3 genes) may be more appropriate than screening for $\mathrm{SHH}$ gene mutations in these patients.

\section{Conflicts of Interest}

The author declares that there are no conflicts of interest regarding the publication of this article.

\section{Acknowledgments}

This work is funded by the College of Medicine Research Center, Deanship of Scientific Research, King Saud University, Riyadh, Saudi Arabia.

\section{References}

[1] S. Malik, "Polydactyly: Phenotypes, genetics and classification," Clinical Genetics, vol. 85, no. 3, pp. 203-212, 2014.

[2] S. A. Temtamy and V. A. McKusick, "The genetics of hand malformations," Birth Defects Original Article Series, vol. 14, pp. 128, 1978.

[3] R. M. Winter and C. Tickle, "Syndactylies and polydactylies: embryological overview and suggested classification.," European journal of human genetics: EJHG, vol. 1, no. 1, pp. 96-104, 1993.

[4] M. M. Al-Qattan, "Preaxial polydactyly of the upper limb viewed as a spectrum of severity of embryonic events," Annals of Plastic Surgery, vol. 71, no. 1, pp. 118-124, 2013.

[5] J. W. Saunders and M. T. Gasseling, "Ectodermal-mesenchymal interactions in the origin of limb symmetry," in MesenchymalEpithelial Interactions, R. Fleichmeyer and R. E. Billingham, Eds., pp. 78-97, Williams \& Wilkins, Baltimore, Md, USA, 1968.

[6] C. Tickle, "The number of polarizing region cells required to specify additional digits in the developing chick wing," Nature, vol. 289, no. 5795, pp. 295-298, 1981.

[7] Y. Yang, G. Drossopoulou, P.-T. Chuang et al., "Relationship between dose, distance and time in Sonic Hedgehog-mediated regulation of anteroposterior polarity in the chick limb," Development, vol. 124, no. 21, pp. 4393-4404, 1997.

[8] K. C. Oberg, J. M. Feenstra, P. R. Manske, and M. A. Tonkin, "Developmental biology and classification of congenital anomalies of the hand and upper extremity," Journal of Hand Surgery, vol. 35, no. 12, pp. 2066-2076, 2010.

[9] A. S. Bauer, M. S. Bednar, and M. A. James, "Disruption of the radial/ulnar axis:congenital longitudinal deficiencies," Journal of Hand Surgery, vol. 38, no. 11, pp. 2293-2302, 2013.

[10] F. Petit, A.-S. Jourdain, M. Holder-Espinasse et al., "The disruption of a novel limb cis-regulatory element of $\mathrm{SHH}$ is associated with autosomal dominant preaxial polydactyly-hypertrichosis," European Journal of Human Genetics, vol. 24, no. 1, pp. 37-43, 2016.

[11] Y. Kawakami, Y. Uchiyama, C. R. Esteban et al., "Sall genes regulate region-specific morphogenesis in the mouse limb by modulating Hox activities," Development, vol. 136, no. 4, pp. 585-594, 2009.

[12] R. Akiyama, H. Kawakami, J. Wong, I. Oishi, R. Nishinakamura, and Y. Kawakami, "Sall4-Gli3 system in early limb progenitors is essential for the development of limb skeletal elements," Proceedings of the National Acadamy of Sciences of the United States of America, vol. 112, no. 16, pp. 5075-5080, 2015.

[13] L. A. Lettice, T. Horikoshi, S. J. H. Heaney et al., "Disruption of a long-range cis-acting regulator for Shh causes preaxial polydactyly," Proceedings of the National Acadamy of Sciences of the United States of America, vol. 99, no. 11, pp. 7548-7553, 2002.

[14] J. E. Vandermeer and N. Ahituv, "cis-regulatory mutations are a genetic cause of human limb malformations," Developmental Dynamics, vol. 240, no. 5, pp. 920-930, 2011.

[15] S. Lohan, M. Spielmann, S. C. Doelken et al., "Microduplications encompassing the sonic hedgehog limb enhancer ZRS are associated with haas-type polysyndactyly and Laurin-Sandrow syndrome," Clinical Genetics, vol. 86, no. 4, pp. 318-325, 2014.

[16] D. Wieczorek, B. Pawlik, Y. Li et al., "A specific mutation in the distant sonic hedgehog ( $\mathrm{SHH}$ ) cis-regulator (ZRS) causes Werner Mesomelic Syndrome (WMS) while complete ZRS duplications underlie Haas type polysyndactyly and preaxial polydactyly (PPD) with or without triphalangeal thumb," Human Mutation, vol. 31, no. 1, pp. 81-89, 2010.

[17] T.-J. Cho, G. H. Baek, H.-R. Lee, H. J. Moon, W. J. Yoo, and I. H. Choi, "Tibial hemimelia-polydactyly-five-fingered hand syndrome associated with a $404 \mathrm{G}>\mathrm{A}$ mutation in a distant sonic hedgehog cis-regulator (ZRS): A case report," Journal of Pediatric Orthopaedics B, vol. 22, no. 3, pp. 219-221, 2013.

[18] K. M. Girisha, A. M. Bidchol, P. S. Kamath et al., "A novel mutation (g.106737G>T) in zone of polarizing activity regulatory sequence (ZRS) causes variable limb phenotypes in Werner mesomelia," American Journal of Medical Genetics Part A, vol. 164, no. 4, pp. 898-906, 2014.

[19] P. Norbnop, C. Srichomthong, K. Suphapeetiporn, and V. Shotelersuk, "ZRS 406A>G mutation in patients with tibial hypoplasia, polydactyly and triphalangeal first fingers," Journal of Human Genetics, vol. 59, no. 8, pp. 467-470, 2014.

[20] C. Vanlerberghe, L. Faivre, F. Petit et al., "Intrafamilial variability of ZRS-associated syndrome: Characterization of a mosaic ZRS mutation by pyrosequencing," Clinical Genetics, vol. 88, no. 5, pp. 479-483, 2015.

[21] M. M. Al-Qattan, I. Al Abdulkareem, Y. Al Haidan, and M. Al Balwi, "A novel mutation in the SHH long-range regulator (ZRS) is associated with preaxial polydactyly, triphalangeal 
thumb, and severe radial ray deficiency," American Journal of Medical Genetics Part A, vol. 158, no. 10, pp. 2610-2615, 2012.

[22] J. E. Vandermeer, R. Lozano, M. Sun et al., "A Novel ZRS Mutation Leads to Preaxial Polydactyly Type 2 in a Heterozygous Form and Werner Mesomelic Syndrome in a Homozygous Form," Human Mutation, vol. 35, no. 8, pp. 945-948, 2014.

[23] M. Sun, F. Ma, X. Zeng et al., "Triphalangeal thumb-polysyndactyly syndrome and syndactyly type IV are caused by genomic duplications involving the long range, limb-specific SHH enhancer," Journal of Medical Genetics, vol. 45, no. 9, pp. 589-595, 2008.

[24] L. Wu, D. Liang, N. Niikawa et al., "A ZRS duplication causes Syndactyly type IV with Tibial Hypoplasia," American Journal of Medical Genetics Part A, vol. 149, no. 4, pp. 816-818, 2009.

[25] J. Sharpe, L. Lettice, J. Hecksher-Sørensen, F. Margaret, R. Hill, and R. Krumlauf, "Identification of Sonic hedgehog as a candidate gene responsible for the polydactylous mouse mutant Sasquatch," Current Biology, vol. 9, no. 2, pp. 97-100, 1999.

[26] T. B. Knudsen and D. M. Kochhar, "The hemimelic extra toes mouse mutant: Historical perspective on unraveling mechanisms of dysmorphogenesis," Birth Defects Research Part C Embryo Today: Reviews, vol. 90, no. 2, pp. 155-162, 2010.

[27] L. A. Lettice, I. Williamson, J. H. Wiltshire et al., "Opposing Functions of the ETS Factor Family Define Shh Spatial Expression in Limb Buds and Underlie Polydactyly," Developmental Cell, vol. 22, no. 2, pp. 459-467, 2012.

[28] R. E. Hill, "How to make a zone of polarizing activity: Insights into limb development via the abnormality preaxial polydactyly," Development, Growth \& Differentiation, vol. 49, no. 6, pp. 439-448, 2007.

[29] C. Tickle, "Making digit patterns in the vertebrate limb," Nature Reviews Molecular Cell Biology, vol. 7, no. 1, pp. 45-53, 2006.

[30] E. Laufer, C. E. Nelson, R. L. Johnson, B. A. Morgan, and C. Tabin, "Sonic hedgehog and Fgf- 4 act through a signaling cascade and feedback loop to integrate growth and patterning of the developing limb bud," Cell, vol. 79, no. 6, pp. 993-1003, 1994.

[31] L. Niswander, S. Jeffrey, G. R. Martin, and C. Tickle, "A positive feedback loop coordinates growth and patterning in the vertebrate limb," Nature, vol. 371, no. 6498, pp. 609-612, 1994.

[32] J. Albuisson, B. Isidor, M. Giraud et al., "Identification of two novel mutations in Shh long-range regulator associated with familial pre-axial polydactyly," Clinical Genetics, vol. 79, no. 4, pp. 371-377, 2011.

[33] H. Li, C.-Y. Wang, J.-X. Wang et al., "Mutation analysis of a large Chinese pedigree with congenital preaxial polydactyly," European Journal of Human Genetics, vol. 17, no. 5, pp. 604-610, 2009.

[34] Z. Zhang, P. Sui, A. Dong et al., "Preaxial polydactyly: Interactions among ETV, TWIST1 and HAND2 control anteriorposterior patterning of the limb," Development, vol. 137, no. 20, pp. 3417-3426, 2010.

[35] Y.-S. Dai and P. Cserjesi, "The basic helix-loop-helix factor, HAND2, functions as a transcriptional activator by binding to E-boxes as a heterodimer," The Journal of Biological Chemistry, vol. 277, no. 15, pp. 12604-12612, 2002.

[36] S. Qu, S. C. Tucker, J. S. Ehrlich et al., "Mutations in mouse Aristaless-like4 cause Strong's luxoid polydactyly," Development, vol. 125, no. 14, pp. 2711-2721, 1998.

[37] D. C. Chan, E. Laufer, C. Tabin, and P. Leder, "Polydactylous limbs in Strong's Luxoid mice result from ectopic polarizing activity," Development, vol. 121, no. 7, pp. 1971-1978, 1995.
[38] J. Charité, D. G. McFadden, and E. N. Olson, “The bHLH transcription factor dHAND controls Sonic hedgehog expression and establishment of the zone of polarizing activity during limb development," Development, vol. 127, no. 11, pp. 2461-2470, 2000.

[39] M. Fernandez-Teran, M. E. Piedra, I. S. Kathiriya, D. Srivastava, J. C. Rodriguez-Rey, and M. A. Ros, "Role of dHAND in the anterior-posterior polarization of the limb bud: Implications for the Sonic hedgehog pathway," Development, vol. 127, no. 10, pp. 2133-2142, 2000.

[40] D. G. McFadden, J. McAnally, J. A. Richardson, J. Charite, and E. N. Olson, "Misexpression of dHAND induces ectopic digits in the developing limb bud in the absence of direct DNA binding," Development, vol. 129, no. 13, pp. 3077-3088, 2002.

[41] A. Galli, D. Robay, M. Osterwalder et al., "Distinct roles of Hand 2 in initiating polarity and posterior Shh expression during the onset of mouse limb bud development," PLoS Genetics, vol. 6, no. 4, Article ID e1000901, 2010.

[42] C.-C. Hui and A. L. Joyner, "A mouse model of Greig cephalo-polysyndactyly syndrome: The extra-toesJ mutation contains an intragenic deletion of the Gli3 gene," Nature Genetics, vol. 3, no. 3, pp. 241-246, 1993.

[43] D. Krawchuk, S. J. Weiner, Y.-T. Chen et al., "Twistl activity thresholds define multiple functions in limb development," Developmental Biology, vol. 347, no. 1, pp. 133-146, 2010.

[44] J. Mao, E. McGlinn, P. Huang, C. J. Tabin, and A. P. McMahon, "Fgf-Dependent Etv4/5 Activity Is Required for Posterior Restriction of Sonic hedgehog and Promoting Outgrowth of the Vertebrate Limb," Developmental Cell, vol. 16, no. 4, pp. $600-$ 606, 2009.

[45] T. Montavon, J.-F. Le Garrec, M. Kerszberg, and D. Duboule, "Modeling Hox gene regulation in digits: Reverse collinearity and the molecular origin of thumbness," Genes \& Development, vol. 22, no. 3, pp. 346-359, 2008.

[46] A. R. Rodrigues, N. Yakushiji-Kaminatsui, Y. Atsuta et al., "Integration of Shh and Fgf signaling in controlling Hox gene expression in cultured limb cells," Proceedings of the National Acadamy of Sciences of the United States of America, vol. 114, no. 12, pp. 3139-3144, 2017.

[47] E. R. Farrell and A. E. Münsterberg, "csall Is controlled by a combination of FGF and Wnt signals in developing limb buds," Developmental Biology, vol. 225, no. 2, pp. 447-458, 2000.

[48] K. C. Oberg, "Review of the molecular development of the thumb: Digit primera," Clinical Orthopaedics and Related Research, vol. 472, no. 4, pp. 1101-1105, 2014.

[49] Y. Litingtung, Y. Li, J. F. Fallon, and C. Chiang, "Shh and Gli3 are dispensable for limb skeleton formation but regulate digit number and identity," Nature, vol. 418, no. 6901, pp. 979-983, 2002.

[50] K. Koshiba-Takeuchi, J. K. Takeuchi, E. P. Arruda et al., "Cooperative and antagonistic interactions between Sall 4 and Tbx5 pattern the mouse limb and heart," Nature Genetics, vol. 38, no. 2, pp. 175-183, 2006.

[51] F. Wang, C. Gao, J. Lu et al., "Leukemic survival factor SALL4 contributes to defective DNA damage repair," Oncogene, vol. 35, no. 47, pp. 6087-6095, 2016.

[52] M. Sakaki-Yumoto, C. Kobayashi, A. Sato et al., "The murine homolog of SALL4, a causative gene in Okihiro syndrome, is essential for embryonic stem cell proliferation, and cooperates with Sall1 in anorectal, heart, brain and kidney development," Development, vol. 133, no. 15, pp. 3005-3013, 2006. 
[53] A. Sato, S. Kishida, T. Tanaka et al., "Sall1, a causative gene for Townes-Brocks syndrome, enhances the canonical Wnt signaling by localizing to heterochromatin," Biochemical and Biophysical Research Communications, vol. 319, no. 1, pp. 103-113, 2004.

[54] J. Böhm, C. Sustmann, C. Wilhelm, and J. Kohlhase, "SALL4 is directly activated by TCF/LEF in the canonical Wnt signaling pathway," Biochemical and Biophysical Research Communications, vol. 348, no. 3, pp. 898-907, 2006.

[55] J. Zákány, M. Kmita, and D. Duboule, "A dual role for Hox genes in limb anterior-posterior asymmetry," Science, vol. 304, no. 5677, pp. 1669-1672, 2004.

[56] B. D. Solomon, "VACTERL/VATER association," Orphanet Journal of Rare Diseases, vol. 6, no. 1, article 56, 2011.

[57] J. H. Kim, P. C. W. Kim, and C.-C. Hui, "The VACTERL association: Lessons from the Sonic hedgehog pathway," Clinical Genetics, vol. 59, no. 5, pp. 306-315, 2001.

[58] P. Stankiewicz, P. Sen, S. S. Bhatt et al., "Genomic and genic deletions of the FOX gene cluster on 16q24.1 and inactivating mutations of FOXF1 cause alveolar capillary dysplasia and other malformations," American Journal of Human Genetics, vol. 84, pp. 780-791, 2009.

[59] M.-M. Garcia-Barceló, K. K.-Y. Wong, V. C.-H. Lui et al., "Identification of a HOXD13 mutation in a VACTERL patient," American Journal of Medical Genetics Part A, vol. 146, no. 24, pp. 3181-3185, 2008.

[60] M. Aguinaga, J. C. Zenteno, H. Pérez-Cano, and V. Morán, "Sonic hedgehog mutation analysis in patients with VACTERL association," American Journal of Medical Genetics Part A, vol. 152, no. 3, pp. 781-783, 2010. 


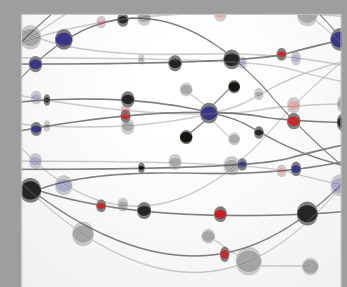

The Scientific World Journal
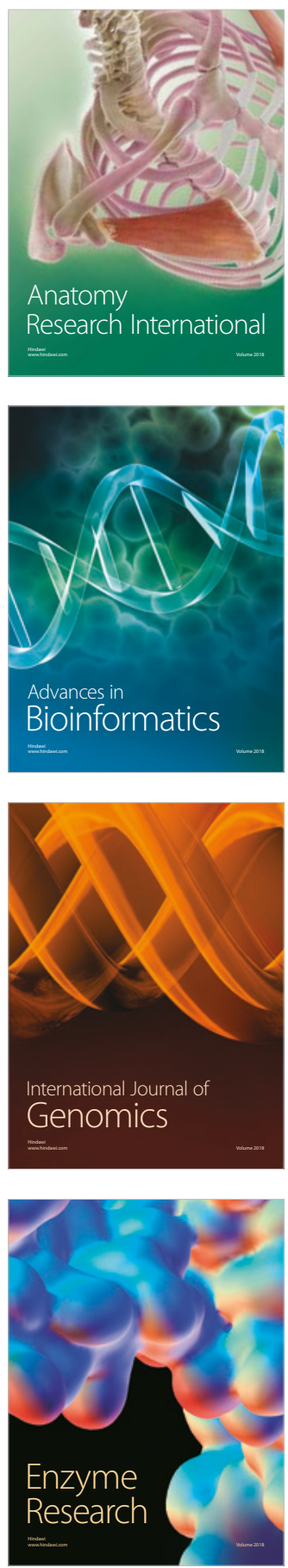
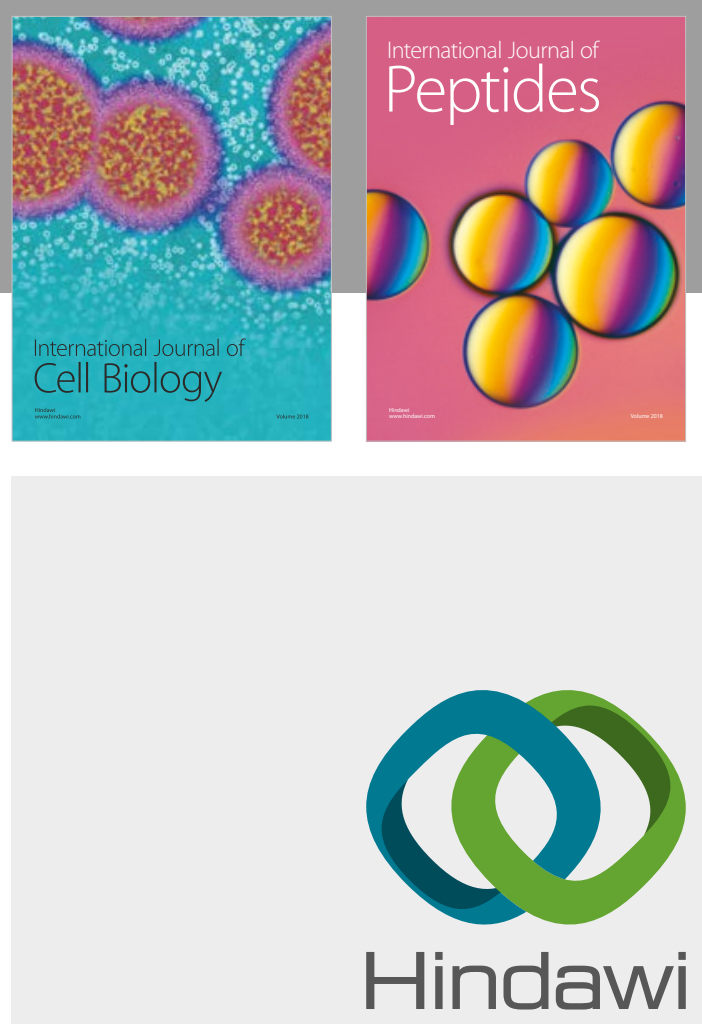

Submit your manuscripts at

www.hindawi.com
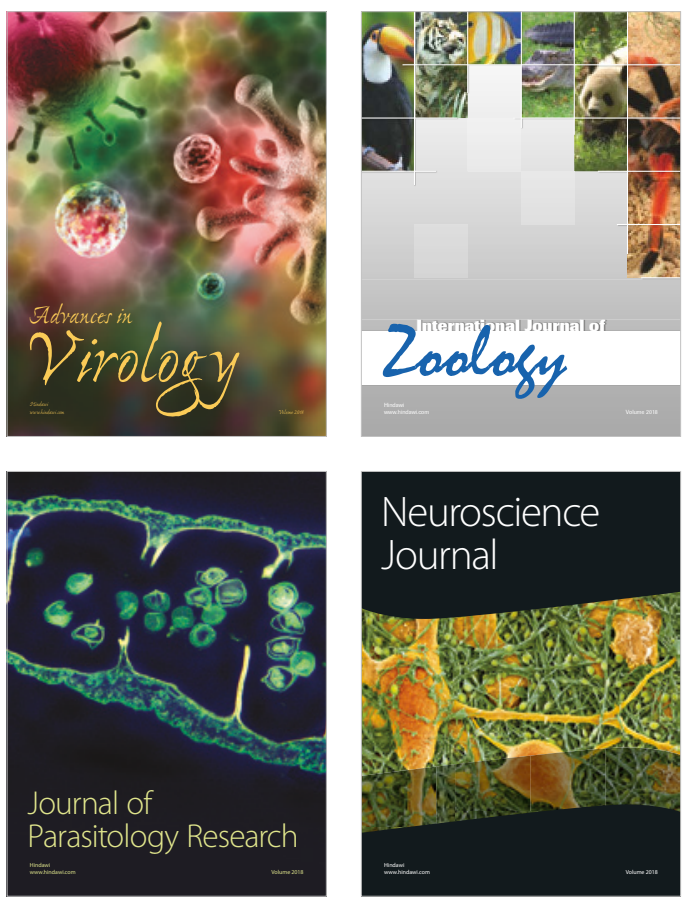
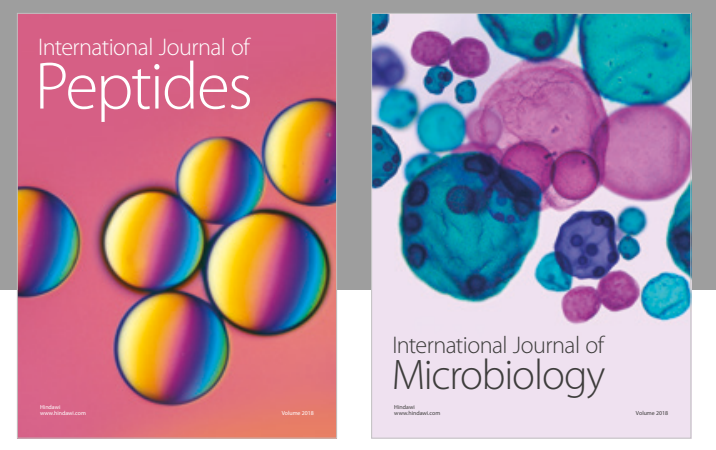

nternational Journal of Microbiology
Journal of
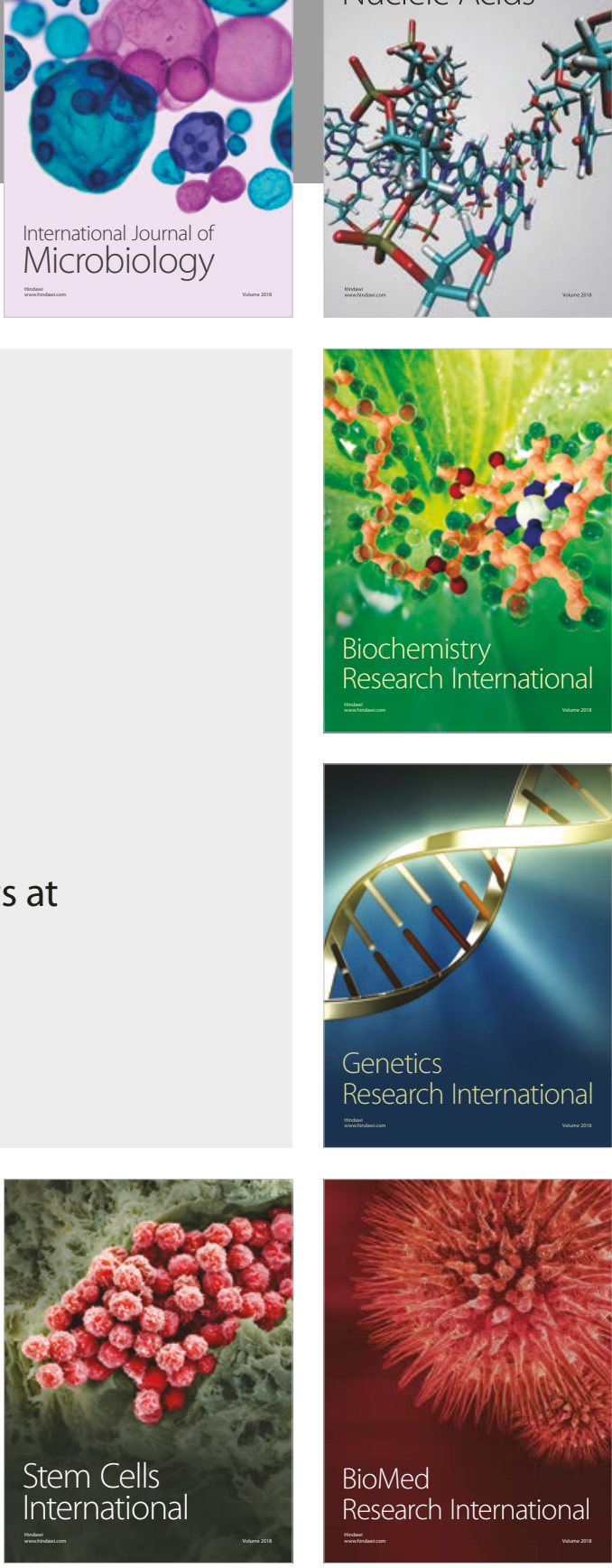
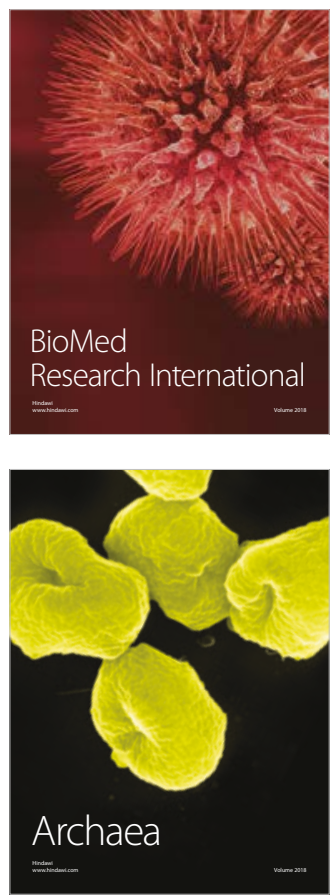